\title{
Recent Developments to Improve the Quality and Economics of Testing for Fatigue and Fracture
}

\section{R. Sunder}

BiSS Research, Bangalore, India

We have developed a number of technologies aimed at rendering test equipment affordable and at the same time reliable and well-supported. These include technologies related to actuator servodrives, energy efficient pumps, modular control and instrumentation electronics and open-source software that is efficient in real-time operation.

Keywords: servo-hydraulic test equipment, power packs, control hardware and software.

Introduction. Several factors govern progress of global industry:

1. Advances in computational techniques and simulation continuously increase operational stress levels in order to reduce weight. This is aimed at improving performance, while at the same time reduce the cost of raw material. Reduced raw material means lower production cost, reduced recycling overheads and increased operational efficiency of the product.

2. Advances in fatigue design improve durability and safety, even at reduced structural weight.

3. Due to globalization, consumer products as well as industrial goods are increasingly sourced from vendors on the promise of acceptable quality at lower prices.

4. As globalization enlarges the number of competitors, time-to-market assumes greater significance as individual firms attempt to be the first to market a new product.

All the above factors demand more widespread use of test equipment to confirm viability of new designs, their quality, performance, safety as well as durability.

Until some years ago, mechanical testing machines were a niche market controlled by a handful of vendors, who could price at will, citing unverifiable costs of developing new and advanced technology. However, the exploding global demand for test equipment has expanded the stage of equipment vendors, with access to advances in digital technology as well as to global partnerships between small enterprises empowered by the Internet as well low cost of movement of people, goods and services.

BiSS is one such developer of test technologies that enjoys a vibrant relationship with partners across the globe. Our objective is to come up with test solutions that are modular, affordable and easily supported, in order that more and more enterprises across the globe can gain access to test technology that until recently appeared unaffordable. The purpose of this paper is to describe a variety of technologies either developed, or, integrated at BiSS Research that contribute to the stated objective. These relate to hydraulics used in universal testing 
machines, grips and fixtures used in testing, control and instrumentation electronics as well as system and application software. The goal is to demonstrate the different means by which test technology and testing itself are being made more affordable to reduce the life-cycle cost of test equipment and thereby empower educational institutions, R\&D and industry to become more productive and competitive in an increasingly globalized framework.

1. Contamination Insensitive Servo-Hydraulics. Fluid power is well entrenched as the most popular means of achieving the widest range of both force variation as well as velocity in mechanical testing. Fluid pumped into a hydraulic actuator causes it to move in the desired direction. The desired force is achieved by building required pressure against specimen resistance. Testing machines based on this principle have been in use from the late 19th century in order to test materials used in machines and structures during the boom of the industrial revolution [1]. The first major transformation in these machines occurred during WW II as electronic force and displacement indicators using strain bridge and LVDT transducers enabled the direct plotting of test results onto $X-Y$ recorders.

In the fifties, William Moog invented what we know today as the two-stage Moog servo-valve [2]. Within a very short span of a few years, this valve became a critical component of aircraft and missile flight control systems. The innovation behind this invention (Fig. 1) was in making it possible for $\mathrm{mW}$ of electrical energy to control many $\mathrm{kW}$ of hydraulic power required to deflect aerodynamic control surfaces such as ailerons, rudders and elevators. Their low mass, combined with the low mass of wiring required to drive them, significantly increased the performance of airborne systems. On the flip side, such valves demanded a contamination free hydraulics system. Even particles as small as 10-15 microns can choke the tiny arteries of the valve's sophisticated pilot stage. The high cost of the proprietary valve, combined with the increased cost of support components such as high-pressure filters were easily justified by the returns in terms of aircraft performance and flight safety.

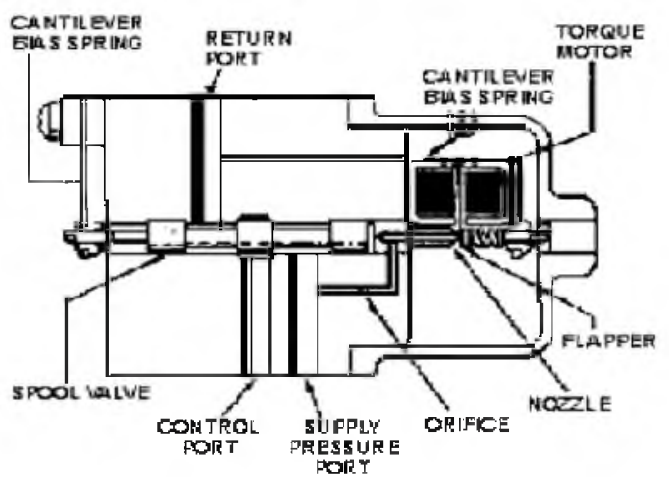

Fig. 1. The dual-stage servo-valve [2]. The remarkable features of this valve are the miniature flapper and microscopic arteries and orifices in the pilot stage that allow high pressure flow control using miniscule currents. This also makes the valve extremely sensitive to oil contamination.

Over the years that followed, the 2-stage Moog servo-valve became an indispensable part of servo-controlled hydraulic systems not only in aerospace applications, but also in surface transportation and industry. These include steel 
rolling mills, wind tunnels, injection molding machines and of course, servohydraulic universal testing machines.

The first servo-hydraulic testing machines appeared in the early sixties. Even though almost five decades have passed since then, the best known manufacturers of testing machines continue to use the same expensive dual-stage servo-valve technology. In the meantime some industrial applications switched to the less expensive and less demanding "direct-drive valves" (DDVs) single stage proportional valves, where electromagnetic force is used to position the flow control spool. The eighties saw the appearance of a new generation of servo proportional valves based on high power rare earth magnets [3]. The performance of these valves is lower than the two-stage valves (Fig. 2), but these are less expensive, more robust and contamination insensitive, making their adaptation to testing machines an economically attractive prospect.

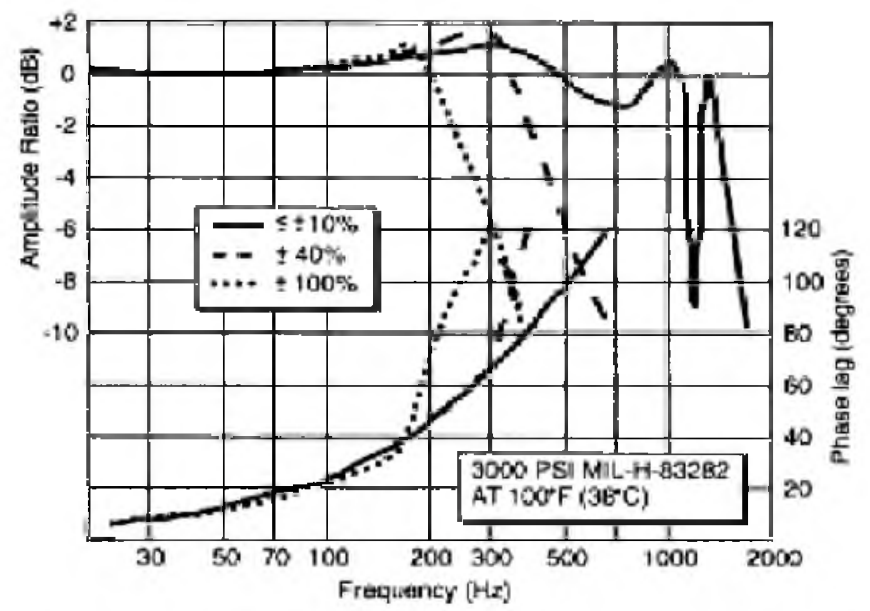

a

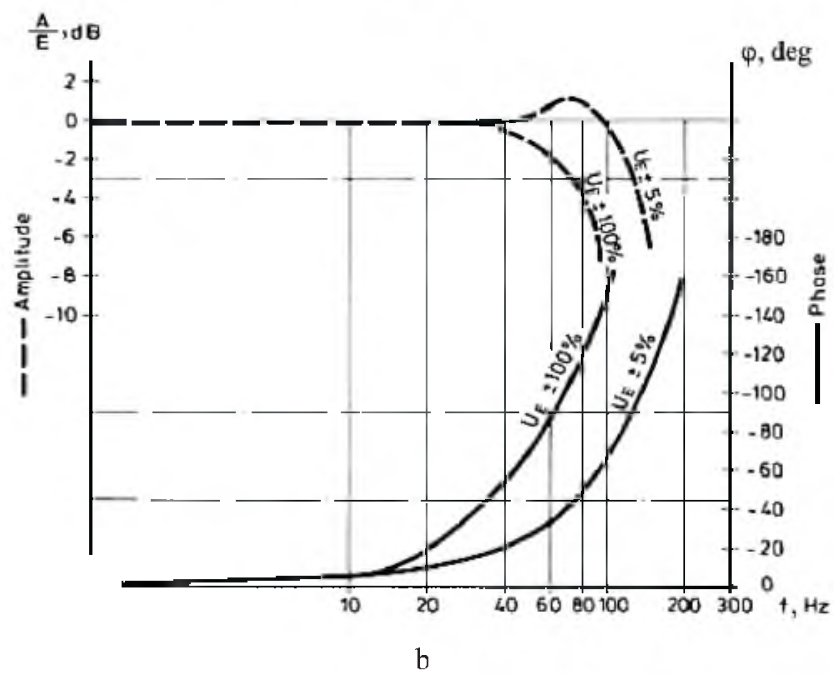

Fig. 2. Performance curves for dual-stage valve (a) [4] and single stage direct-drive valve (DDV) [5]. While the dual stage valve can operate at frequencies up to a few hundred $\mathrm{Hz}$, the DDV (b) is effective only up to about $100 \mathrm{~Hz}$. Most cyclic test requirements seldom demand more than $50 \mathrm{~Hz}$. 
In the early nineties, BiSS Research developed its first servo-actuators with DDVs. With suitable changes to electronics and hydraulics, these valves were found suitable for fatigue cycling at up to $100 \mathrm{~Hz}$ and in terms of precision control, even superior to the two stage valves because of their reduced hysteresis and improved linearity in flow control. Moreover, as they are virtually insensitive to contamination, high-pressure fine filtration is not required, rendering the solution less expensive to install and maintain and at the same time, more reliable. Multi-stage servovalves using DDVs at the pilot stage deliver several hundred LPM of flow and enable test velocities as high as $7 \mathrm{~m} / \mathrm{s}$. BiSS experience with these valves extends from low force actuators down to $5 \mathrm{kN}$ and as high as $1000 \mathrm{kN}$. One may note that none of these applications would have exhibited improved performance with dual-stage valves given their end-use. The application of DDVs has reduced the cost of test equipment by comparison to that of brand names that continue to use the older dual-stage technology. What is more, even in the absence of pressure line filtration, for over fifteen years now and about a million hours of cumulative usage experience, there have been no reported cases of valve failure. This indicates why DDVs are preferred in terms of life-cycle cost as well.

In summary, DDVs make test equipment more affordable by the use of fewer and less expensive components on a test system, without compromising the performance and quality requirements of the test process. They also reduce operational costs and increase system reliability.

2. Variable Speed Pumps. Hydraulic power is usually delivered by gear pumps, vane pumps, radial or axial piston pumps. The first two are fixed flow, while the last two may be made variable flow through the inclusion of a swash plate that controls piston stroke. Piston pumps are typically much more expensive than gear or vane pumps and variable flow technology makes them even more expensive. Typically, at flow rates less than 80 LPM variable delivery pumps are seldom used as they turn economically unviable. Most universal test systems require pump capacity between 4 and 65 LPM. Therefore most such machines use fixed delivery pumps, unless multiple test systems are connected to the same pump, justifying a larger capacity variable delivery option.

The pump is usually selected to meet the highest sustained performance requirement of the servoactuator. Assuming that over most of its usage, the machine will be operated at speeds that are much less than its required peak performance, much of the power from the pump will be dissipated as heat as excess high pressure flow is released through the relief valve. Apart from the energy wasted in this process, more energy needs to be spent on cooling the heated oil. These make most conventional servo-hydraulic test systems rather inefficient and therefore more expensive to operate. On an average as high as $80 \%$ of the connected power may be wasted as heat. Thus, on a 40 LPM pump, as much as $15 \mathrm{kVA}$ of consumed power may be dissipated as heat. This leads to the need to have large, closed loop cooling systems that are expensive to install and also consume another 2-3 kVA of power.

Some eight years ago, BiSS Research developed the concept of servocontrolled pumps for its range of digitally controlled universal testing machines. Their development is made possible by the appearance in the market of so called 
variable frequency drives (VFDs). When combined with a suitably designed servo-circuit that uses system line pressure as feedback, along with a set of safety limit interlocks to protect both the drive as well as the pump and motor assembly, one can achieve constant pressure on the system through automatic regulation of pump r.p.m. Thus, the pump now consumes only as much power as is required to meet the instantaneous hydraulic flow requirement on the servoactuator.

Over the years, BiSS has designed VFD driven pumps with flow rates ranging from 4 to 400 LPM. Industry feedback suggests that they save up to $70 \%$ in consumed energy, reduce cooling requirements and are also a lot quieter than conventional pumps. Figure 3 shows an example of these pumps and their drives. As will be shown later, smaller 4 LPM pumps require no cooling at all - the limited amount of heat generated on the system is dissipated by the exposed sides of its enclosure.
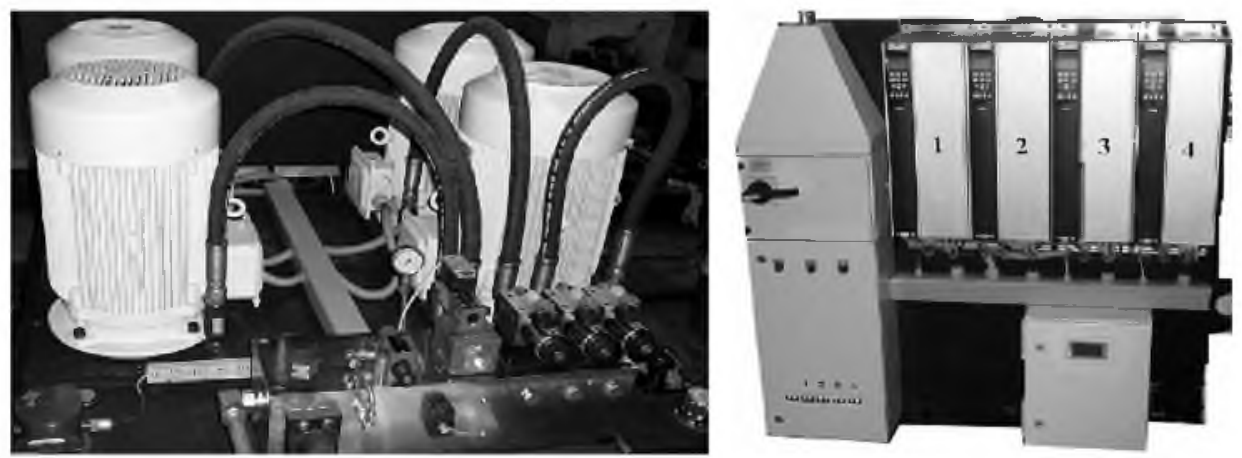

Fig. 3. $4 \times 65$ LPM fixed delivery pumps (left) driven by four $30 \mathrm{kVA}$ variable frequency drives (VFDs). The programmable logic controller (PLC) controlling the four VFDs ensures that only that fraction of installed $120 \mathrm{kVA}$ that is really required is used at any given instant. This results in considerable reduction in energy consumed and also in energy dissipated as heat. The cooling system for this power pack is comparable to that used in conventional pumps of considerably lower capacity. Use of commercially available off-the shelf (COTS) components such as PLCs and VFDs ensures easy maintenance with readily available spares.

In summary, the technology of servo-control on pump motor speed makes servo-hydraulic test systems considerably more efficient than ever before. This "green" solution also provides many other benefits such as reduced operational cost, lower noise in operation and possible increase in pump life. Also, measured pump parameters can serve condition-monitoring purposes.

3. Self-Aligning Low-Cycle Fatigue Grips. Low-cycle fatigue (LCF) testing demands the capability to enforce tension-compression loading far beyond the theoretical buckling load of the specimen. Without this capability, one can neither obtain the cyclic stress-strain curve for a material, nor determine fatigue properties under conditions of cyclic plastic strain (see Fig. 4a).

LCF testing demands near-perfect alignment of the load train to ensure the absence of bending component from off-center loading. ASTM E 606 prescribes a $5 \%$ limit on bending stresses. To meet this requirement, conventional test systems are supplied with an alignment kit. The kit includes instruments to measure coaxiality and colinearity of the top and bottom gripping on the test system and an alignment correction unit placed between the top crosshead and load cell that 
permits controlled shifting of the top axis as well as tilt correction if required. The kit also includes an instrumented specimen that can sense dual-plane bending moments arising from potential misalignment, along with the electronics to condition the transducer outputs and the data acquisition to measure and display quality of alignment.
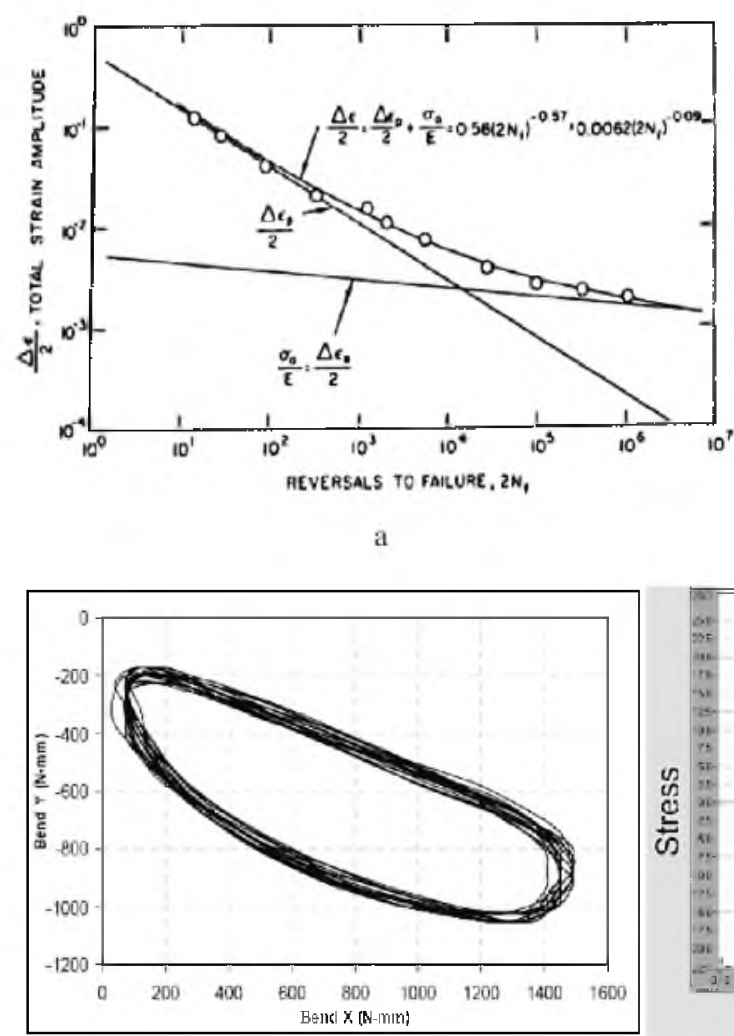

$\mathrm{c}$

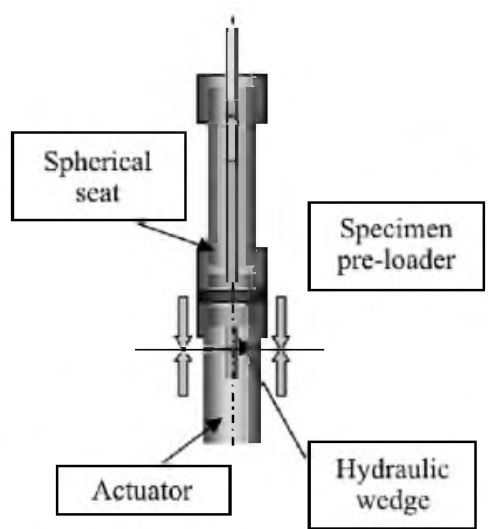

b

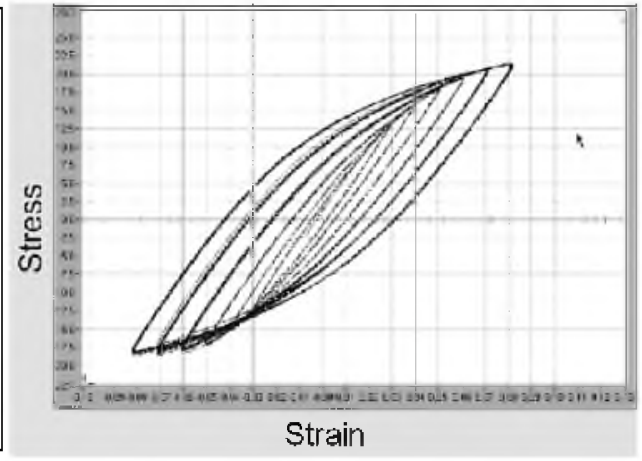

d

Fig. 4. (a) LCF data require tension-compression testing under cyclic plastic strain. Excellent specimen alignment is required to make this possible. (b) Schematic of hydraulic self-aligning grips developed at BiSS Research. The hydraulically locked spherical seat eliminates specimen backlash and bending moment at start of test. Hydraulic wedge locks grip to actuator at bottom and load cell at top. (c) Typical $\mathrm{X}$ and $\mathrm{Y}$ bending moment during cycling at $\pm 10 \mathrm{kN}$ load using self-aligning grips. Bending stress from these moments are les than $5 \%$ of applied stress, which meets the requirements of ASTM E 606. (d) Typical cyclic stress-strain response with self-aligning grips. Note the absence of zero-crossover glitch and symmetry of loading/unloading response.

Once a system is aligned using the above procedure, coaxiality is unlikely to change because the rigid fixtures are preloaded and locked. However, any movement of the crosshead followed by reclamping may introduce angular misalignment. Further, system alignment by itself may not serve as a guarantee of specimen alignment. Specimen axiality and threading tolerances may introduce variations in alignment between specimens, that the above alignment procedure is not in a position to address.

Figure $4 \mathrm{~b}$ describes an engineering solution developed at BiSS Research that attempts to address the demands of specimen-to-specimen realignment. Both the 
top and bottom grip consist of three important elements. A hydraulic lock is provided between grip and actuator/load cell to ensure backlash free grip mounting. A spherical seat on the specimen pull rod ensures both angular and lateral axis re-alignment of each individual specimen through tensile cycling at small loads. A hydraulic press with pressure control acts as a hydraulic lock on the specimen and spherical seat in order to guarantee zero-backlash rigid response in tension-compression loading.

Figure $4 \mathrm{c}$ shows the results of random alignment checks using an instrumented specimen. Without exception, bending stresses appear to be well within prescribed norms. In addition to the advantage of a lower cost solution that avoids the need for additional fixtures and instrumentation, the self aligning grip offers a few other advantages that are noteworthy. It can be readily converted into a high temperature version by using temperature resistant pull rods. Mounting and dismounting the grip or the specimen is much faster than on conventional grips. And finally, the solution guarantees alignment of each new specimen, irrespective of its own dimensional variations. This is because in tension-tension cycling at small load, those very moments that are caused by misalignment work towards re-alignment of the spherical seat.

Figure $4 \mathrm{~d}$ shows typical cyclic stress-strain response under incremental strain cycling.

4. "Gauge Free" Displacement Measurement. All analog transducers share a common shortcoming. Their measurement precision is determined by gauge length. Thus a $0.1 \%$ precision on a gauge length of $10 \mathrm{~mm}$ would mean error of 10 microns, while on a gauge length of $100 \mathrm{~mm}$, it would be 100 microns.

It follows from the above that quality test results require that transducer ranges need to be not much greater than what is required for a given test. Obviously, if fatigue cycling needs to be performed between 2 and $4 \mathrm{kN}$, a $10 \mathrm{kN}$ test system will ensure one-tenth the error one would observe on a $100 \mathrm{kN}$ test system.*

All servo-hydraulic test systems are equipped with LVDTs on the actuator that provide stroke readout. These are typically between \pm 50 and $\pm 150 \mathrm{~mm}$ range. Errors in stroke readout would thus be in the region of about 100 microns, which is quite unacceptable when testing specimens for modulus or fracture mechanics. These tests require additional clip-on or non-contact transducers that measure displacement over specimen gauge length. Thus, extensometers are used in tension and LCF testing, while COD gauges are used in fracture mechanics testing. The error in such transducers is of the order of only a few microns because the displacement range is only a few millimeters.

Room temperature transducers are affordable. However, high temperature extensometers and COD gauges are not only expensive, but also delicate and therefore prone to damage in repeated usage. If it were possible to use the stroke transducer to obtain quality readouts of specimen gauge length displacement, the cost and effort of testing would be considerably reduced. Also, this would make

\footnotetext{
* One needs to note in this regard that any attempt to sub range the transducer either through amplification in electronics or data acquisition will only improve resolution. Noise and drift will increase proportionately with signal amplification.
} 
testing in aggressive environments possible as would be the case with corrosion bath and also with irradiated specimens that pose a safety hazard in gauge mounting.

As mentioned earlier, the stroke range in test systems is one or two orders of magnitude greater than that of the displacement range required in most mechanical tests. The other problem associated with a "remote" measurement such as stroke is its sensitivity to the displacement response of the test system as a whole. As shown in Fig. 5, application of load will cause deformation of not only the test specimen, but also the load frame as well as the load train including actuator rod and load cell, pull rods, grips and the several fasteners holding them together. The latter also introduces a non-linear element into the stroke versus load relationship.

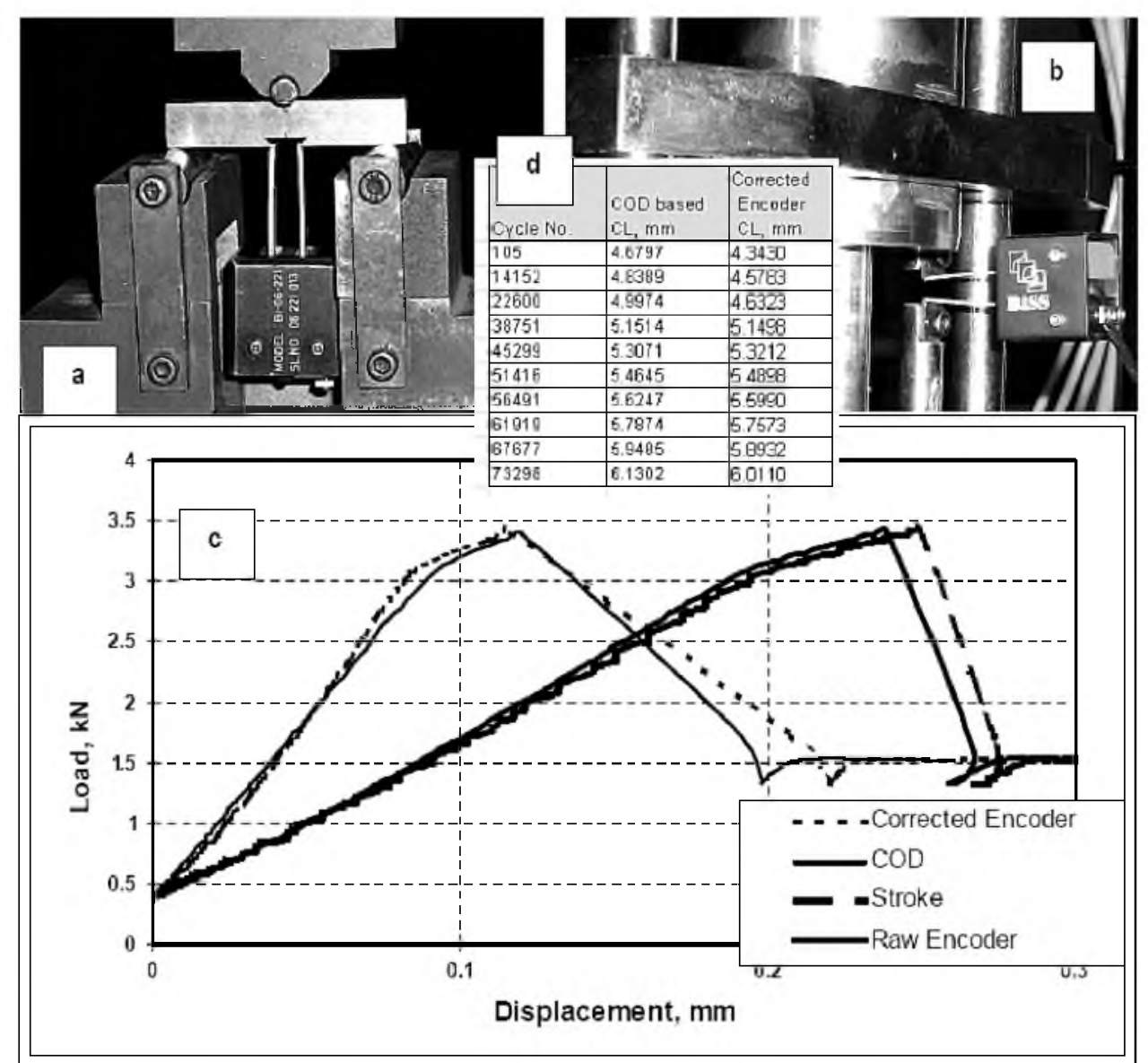

Fig. 5. (a) COD gauge mounted on $\mathrm{SE}(\mathrm{B})$ specimen as required by relevant test standards. The gauge provides accurate measurement of crack opening displacement, but is unsuitable for aggressive environments that may damage the gauge. (b) COD gauge mounted using special fixture to accurately measure actuator displacement (stroke). A digital linear encoder may also be used for the purpose. This location is away from the specimen area and is immune to test environment. (c) Specimen COD response compared with values deduced (corrected) from remote measurement using linear encoder on actuator rod. Similar data would be obtained from gauge mounted as shown in (b). (d) Crack size computed from specimen COD and from actuator position inputs. Note the similarity in estimates, suggesting viability of alternate measurements. 
Assuming a fixed crosshead position and assuming all the load train components are tightly fastened, one may expect the stroke versus load relationship to be reproducible even if non-linear. This still leaves the problem of stroke precision which may be resolved either by using a shorter range displacement transducer, or, by resorting to a state-of-the-art digital encoder based measurement of stroke that guarantees resolution and precision that is independent of stroke range. Presented below are results from attempts to use both approaches. The experiments were performed with 3-point single-edge bend (SE-B) specimens tested in fatigue pre-cracking followed by fracture test.

Figure 5 shows test results using a 0.1 micron digital encoder measuring stroke. Similar results were obtained using a COD gauge mounted on the servo-actuator to measure its movement (Fig. 5b). In both cases, an extremely stiff un-cracked dummy specimen was used to obtain the stroke versus load relationship. Then, during testing with the real specimen in place, stroke computed as a function of load from this relationship was subtracted from the stroke readout in order to obtain specimen related displacement.

From the results in Fig. 5c, d, one may summarize that reasonable estimates of specimen response can be obtained from actuator stroke measurement. The quality of these measurements is determined by precision of the remote measurement as well as the reproducibility of the stroke versus load response for the dummy (stiff) specimen. It appears desirable that a standard practice be evolved to permit valid industrial quality tests without having to use gauges on the specimen. This would reduce the cost of testing and perhaps also improve productivity. And it would significantly improve productivity in test labs associated with irradiated specimens such as in nuclear reactor safety assurance.

5. Processor-Independent Modular Hardware. Servo-hydraulic test systems find applications in the most diverse areas. Typical examples include materials test systems to determine strength and durability of materials, high performance testers for elastomers, low rate systems for stress-corrosion, cyclic creep and LCF, moderate force high velocity test systems for suspension components, biaxial test systems including axial-torsion systems, single and multi-channel structural test systems using several actuators and tightly coupled high performance test systems such as Stewart Table (6 actuators) and earthquake simulators (8 actuators). Control hardware and software are the heart and soul of all these systems. This raises the question about how to modularize both the hardware and software such that a collection of common modules can meet all of the above requirements.

Modularization is important for several reasons. Use of common hardware and software components across the wide variety of applications described above implies reduced production as well as spares support cost due to volume and common inventory considerations. It means that hardware technicians are invariably familiar with hardware set up, support and troubleshooting because it does not differ between applications. Finally, if it is indeed possible at all to have common software modules between applications, it can have a favourable impact not only on the cost of application software, but also on the quality of customer support, not to mention ease of migration for the user from one application to another. In the pursuit of such goals, the developer needs to ensure that individual modules seldom contain features that are redundant to many users. Failure to do 
so would escalate costs for users who end up paying for something they do not have use for.

In an attempt to modularize both hardware and software to meet the above objectives, BiSS Research came up with a few hardware and software solutions, which over the years have shown a noticeable degree of success. A motherboard with components required on most test equipment along with six plug-in slots for add-on cards was developed as a common hardware platform for all the applications listed above. A combination of six different add-on cards were developed such that a suitable mix of these cards can address all of the applications listed above. These are essentially digital signal conditioning and data acquisition cards to handle different transducers used on testing machines, including LVDTs, load cells and other strain bridges, encoders and accelerometers. With suitable packaging of the cards and connectors, up to sixteen independent data acquisition channels along with signal conditioning can be connected to a single card, expanding instrumentation channel count capacity to over 100 on the controller. Controller firmware and application software were suitably designed to handle all of the combinations that may arise from the hardware modularity. Finally a number of software modules were developed whose functionality is not affected by channel count or hardware configuration. These include log mode synchronous data acquisition across up to 150 channels at speeds of up to $100 \mathrm{kHz}$ (cumulative), multi-channel control and command waveform generation from one to 32 channels, and multi-panel, multi-channel real-time graphics to display collected real-time data.

6. Single Footprint Test Systems. Conventional perception of a servohydraulic universal test system is that of separate load frame, hydraulic power pack and control console. Not readily apparent in the scheme are the need for three phase power supply and water or air cooling utilities to support system operation.

As shown in the previous section, energy efficient servo-controlled pumps dissipate less heat and are less noisy. Low performance test systems such as LCF test systems and lower force rating test systems, typically $25 \mathrm{kN}$ and less, require smaller pumps. Obviously, such pumps will dissipate even less heat, raising the possibility of completely doing away with active cooling and its associated cost and energy consumption. On the other hand, newer pump assemblies that have appeared in the market are extremely compact and competitively priced, thanks to the pressure die casting technology that permits integration of several hydraulic components into a single compact manifold.

Figure 6 shows how the above technologies permit the integration of the load frame, pump and console into a single footprint scheme. The load frame itself uses hollow pipe columns that are light weight and also act as conduits for sensor cables. High strength Al-alloy crosshead and platen coupled with these columns make a stiff load frame rated to $25 \mathrm{kN}$ dynamic capacity.

The bottom platen of the load frame is at an elevated height to suit the convenience of the operator. The enclosure below the platen houses the servoactuator, hydraulic power pack as well as the controller. The sides of the enclosure act as radiators to cool the oil. The entire system weight is below $175 \mathrm{kgs}$ and is packaged for air shipment. It operates at ambient temperatures up to $35^{\circ} \mathrm{C}$ and 


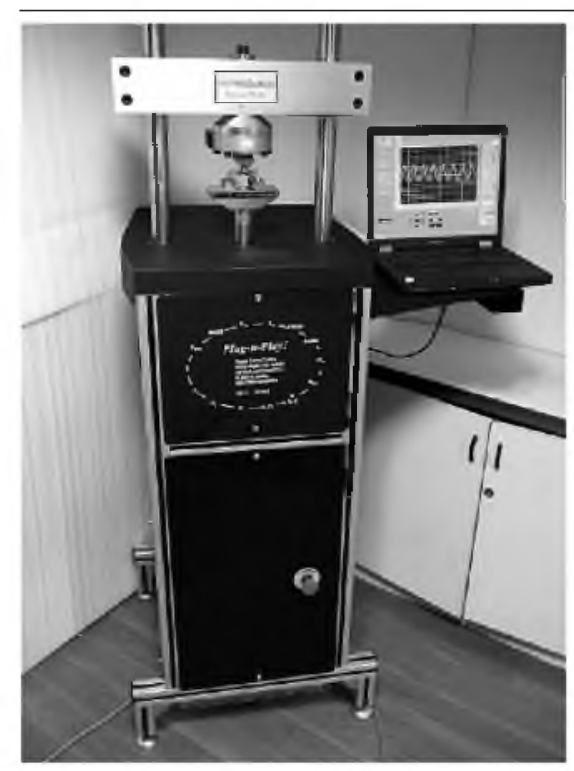

Fig. 6. Fully integrated standalone digitally servocontrolled test system, suitable for tension/compression. fatigue and fracture testing. Once plugged in to a $220 \mathrm{~V}$ mains socket, the system is ready to use. The load frame is rated to $25 \mathrm{kN}$. The hydraulic power pack and digital controls are located in the lower half of the enclosure. The system is connected to any industrystandard notebook or desktop PC. With force rating between 10 and $25 \mathrm{kN}$ and frequency response up to $100 \mathrm{~Hz}$, the system may be used to perform most materials testing that is normally carried out on much larger conventional test systems. Heat dissipation from the side walls of the enclosure is adequate to cool the power pack. Weighing in at under $200 \mathrm{kgs}$, the system may be readily relocated for demonstrations in a lecture hall or seminar room.

draws single-phase $220 \mathrm{~V}$ mains supply. It is suitable for tension/compression testing as well as for fatigue and fracture testing at up to $100 \mathrm{~Hz}$.

Systems such as the one in Fig. 6 appear to change the way one sets up a testing laboratory, or even how one budgets experimental research. Unlike conventional test systems, they do not require special flooring or utilities such as three-phase mains supply, special grounding and water cooling, not to mention access and material handling equipment that is demanded by heavier machines. Finally, the absence of external cabling and disappearance from view of oil and water hoses makes the system an unobtrusive and less conspicuous part of the laboratory premises, much like other office equipment. The aesthetics of this solution combined with its reduced life-cycle cost were made possible by several recent advances in technology.

7. Integrating Large Channel Count Instrumentation with Test Control. Most mechanical tests involve the use of standard transducers to measure gaugelength displacement, applied force, etc. However, researchers sometimes wish to strain gauge a test specimen at different locations, or, to measure local displacement or deflection using a number of gaging LVDTs. As conventional controllers of testing machines do not allow too many data acquisition channels, a dedicated strain gauge or displacement data acquisition is acquired to meet instrumentation requirements. Being a niche market, these data acquisition systems can cost as much as the test controller itself. And the user ends up with the need to set up and operate a second system, probably connected to a separate computer system and working with a different application program. At the commencement of the test, the user carries the added responsibility of wiring machine readouts such as force and stroke to the data acquisition unit, so that reference readouts are available against the logged data. Finally, there remains the problem of controlling both the systems through the test in order to ensure that not only the test proceeds successfully, but also, that all useful data are logged on the second system in a manner that can be used to reconstruct specimen response. The modular control 
hardware and software described earlier attempts to address the need to incorporate large channel count instrumentation into the test system control hardware and software for the convenience of the end user and for the affordability of the solution. With this system, user can log tens of strain and LVDT outputs along with the command waveform, stroke and load readouts, without the need for an expensive dedicated data acquisition unit. For example, a large panel subject to cyclic loading under complex load sequences can be strain gaged at a number of locations along the path of a growing fatigue crack in order to study strain distribution under cyclic conditions as well as their redistribution under subsequent static loading to fracture (see Fig. 7).
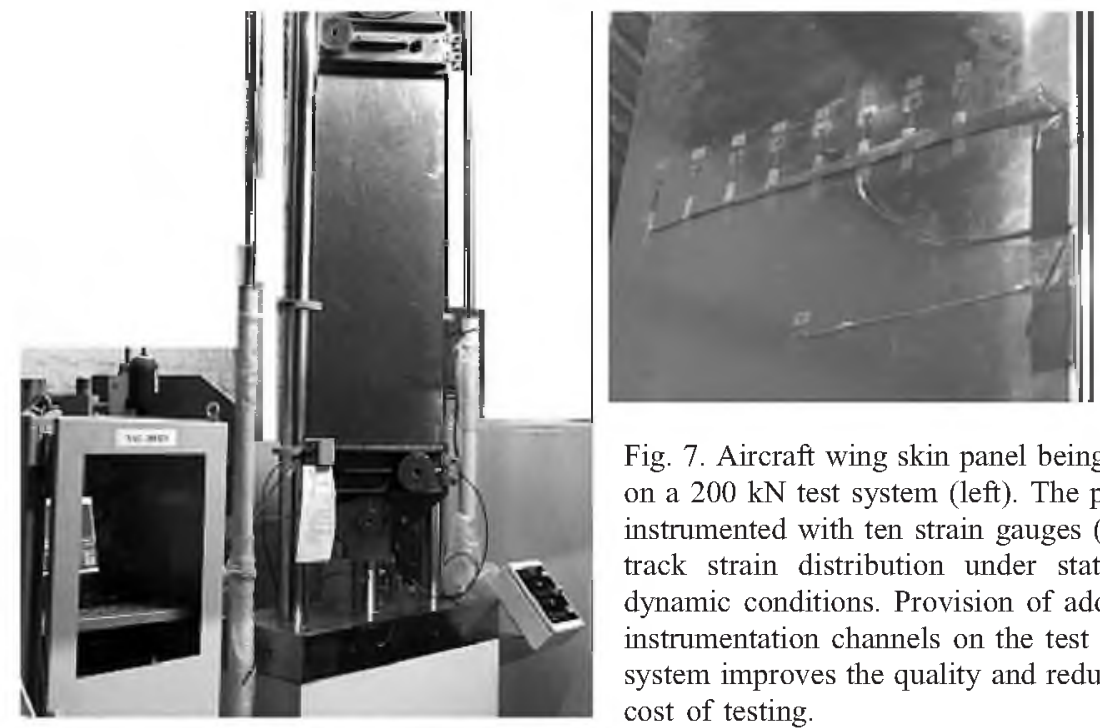

Fig. 7. Aircraft wing skin panel being tested on a $200 \mathrm{kN}$ test system (left). The panel is instrumented with ten strain gauges (top) to track strain distribution under static and dynamic conditions. Provision of additional instrumentation channels on the test control system improves the quality and reduces the cost of testing.

8. Global Data Sharing. Consider the prospect of having to "oversee" the quality of test control performed by a particular application. An example would be the monitoring of how accurately, the application program maintains the mean and amplitude of cycling in a long duration fatigue test, so that a qualification certificate based on real measured data may be issued. With most conventional test software, one would have to be reconciled with the possibility of accepting the vendor's process of performing the test and collecting test results. Independent verification of actual loads applied would be effectively ruled out, unless an attempt is made to connect an additional load cell or at least a data acquisition system for independent measurement of applied force versus time.

The problem with most "canned" software is that the user has no means of observing in "real-time", the data that flow through the controller and computer system. Most testing machines using host computers rely on the MS-Windows platform. With the advent of 32-bit memory protection starting with Windows 98 and NT operating systems (OS), multiple tasks cannot share common data, because memory space belonging to a particular task cannot be accessed by another task. Data sharing under MS-Windows is implemented by copying data to an intermediary, usually a resource on the OS, which in turns passes it on as "legal tender" to the target application (see Fig. 8a). This is how applications, 
even those that form part of MS-Office share data. This may serve the purpose of memory protection against corruption by other tasks and the associated overheads may go unnoticed in conventional operations associated with PCs. However, the scheme presents serious shortcomings with regard to real-time application. Considerable slowdown and memory "hogging" can occur as multiple copies of large amounts of data are passed around to different participating tasks (Fig. 8a). As these are real-time data, there is no guarantee that all participating tasks "see" the same data at the same time. Thus a load readout may not "appear the same" at a given time across multiple tasks and give rise to conflicting decision making among tasks.
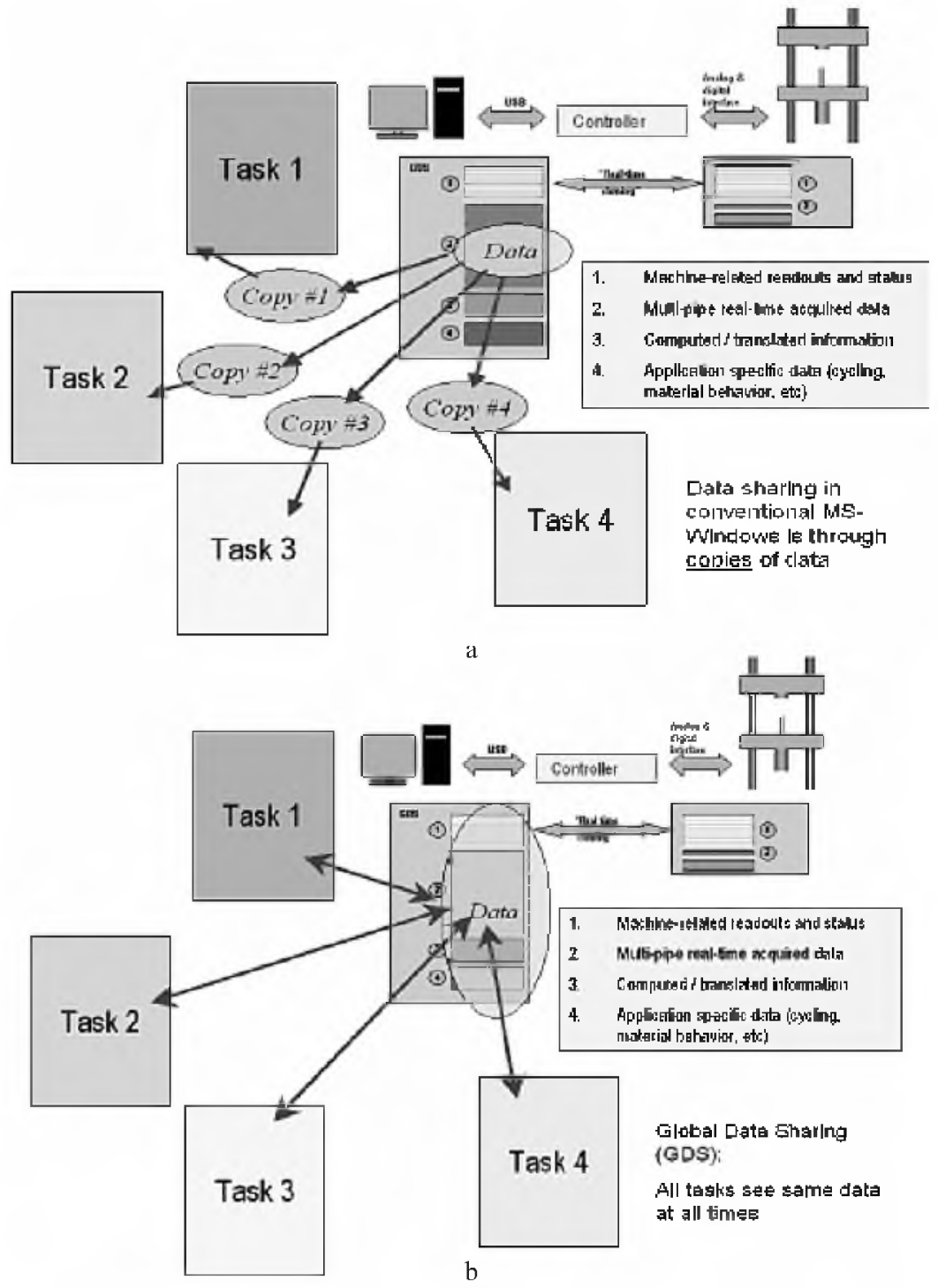

Fig. 8. (a) Conventional data sharing between concurrent tasks in the MS-Windows considerably slows down real-time multi-tasking. This is the operational mode of the well known Windows data sharing mechanisms such as dynamic data exchange (DDE) and ActiveX controls. (b) The GDS environment provides for concurrent tasks to directly access the same physical address space. This considerably speeds up real-time computing. 
In an attempt to regain the advantages of multi-tasking without compromising synchronization and throughput of real-time data flow among tasks, a data structure and access scheme was implemented within the framework of MSWindows that protects the integrity of the application and at the same time eliminates the need for data messaging (see Fig. 8b). We call it global data sharing, or, GDS. All participating tasks directly and concurrently operate on both static as well as dynamic areas of a so-called virtual machine (VM). The contents of the VM appear as arguments in individual tasks, so much so, that when a value is changed by one of the tasks, all other tasks will instantaneously "see" the new value - even if one or more of them is in debug or trace mode.

Dynamic data are in the form of circular buffers carrying incoming or outgoing data from/to the controller. The buffers are large enough to carry data covering over a $100 \mathrm{~s}$ of transfer. This means that the different tasks may access them asynchronously without affecting their integrity. It also means that if the OS briefly hangs due to time critical operations on the disk or on the network, the real-time data flow even if interrupted, will not result in lost data.

Up to 64 independent tasks can "register" for access to GDS. The access process ensures that tasks unrelated to the concerned application cannot corrupt the GDS. It also enables the operation of more than one GDS. Thus, it is possible for multiple test systems to be connected to a single host computer and for multiple sets of the same or different application to hook up to individual GDSs. Work is in progress to provide GDS access across the network and also on the Internet. This opens the possibility of developing laboratory management software that can hook up to multiple test systems. It will also open the potential of remote test control. A major advantage of GDS is that it is software platform independent. Thus, code developed on a variety of development platforms can work together, allowing for end-users and developers alike to choose the platform of their choice to control the test system. This has been confirmed through sample real-time applications written in Visual C++, VB, MS-Office Macros, Lab-Windows, LabView and Delphi. As exclusivity threatens the prospect of cost escalation without necessarily improving quality, user-access to the real-time application introduces an element of security and control.

9. Open Source MS-Office Interface. An overwhelming majority of professionals use computers with MS-Windows. At the workplace, they rely on MS-Office to perform their day-to-day documentation work. This includes MSExcel (XL), which permits reasonably complex processing, arrangement and storage of vast amounts of data. XL provides for data representation in user -definable report format. It also incorporates the VB-Macro (VBM) facility, whereby, user-written code in VBM can process and store information in the XL Worksheet. The GDS environment described above permits XL-based VBM applications to set up and control a test and also collect, process and store test data in real-time. Such an application can be created to perform any particular test however complex it may appear. Figure 9 shows a typical user interface for an XL-based fatigue crack growth test application.

The XL-file thus stores the test assignment including operator and customer details, collected data, processed results as well as the test report. In addition, the same file also contains the VBM code that was used to execute the test and 
process results. This guarantees a degree of traceability that may not have been possible without such a file structure and not to mention without GDS. Finally, if the code is left unprotected, it becomes open source and therefore amenable to change, improvement as well as exchange within the testing and research community.

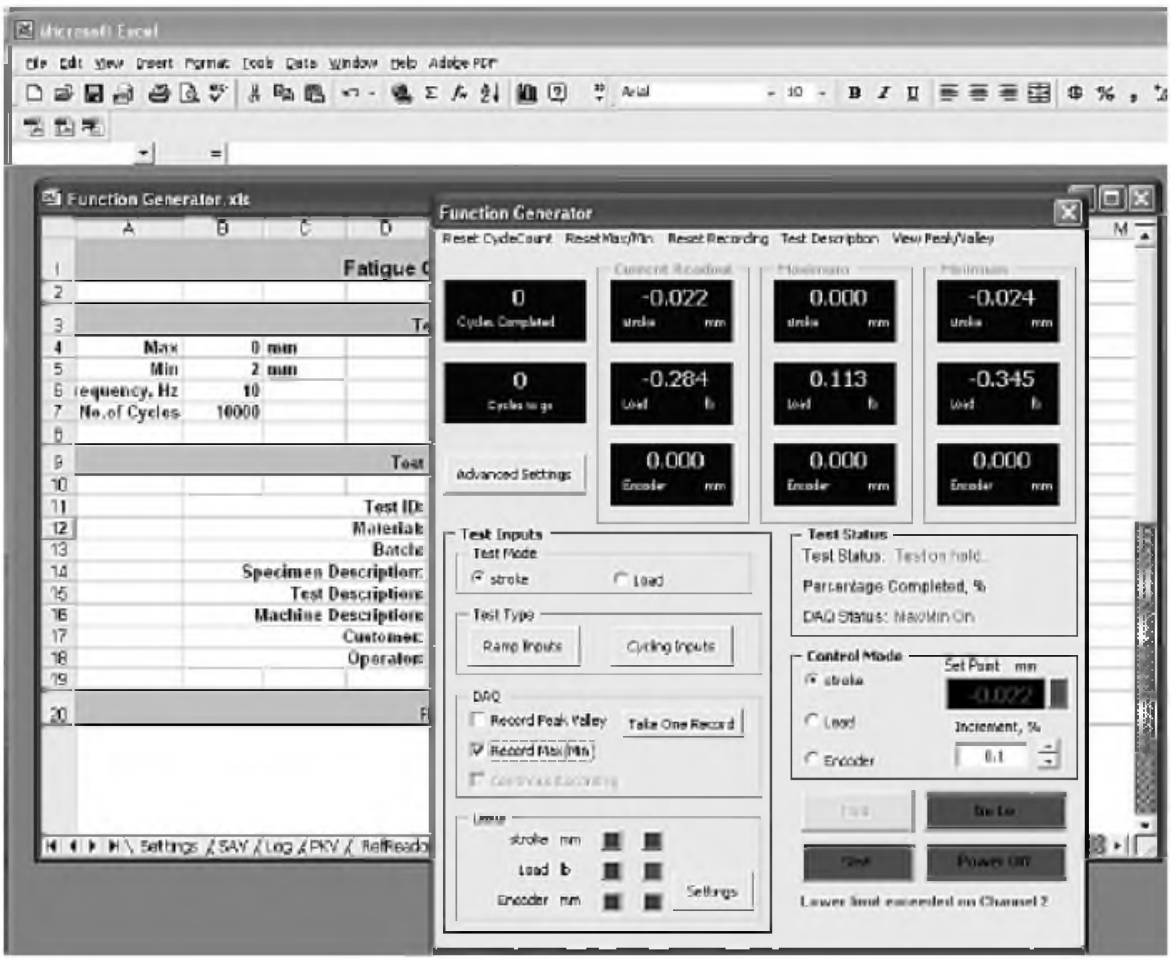

Fig. 9. Typical user interface of an XL-based real-time application written as VB macros. Real-time commands to perform test control as well as data acquisition are embedded in the XL-file as open-source code. Data are written directly into the desired XL worksheets. The end user is free to process the data in a different way if desired either manually, or, by writing new macros. The source code can also be changed if required to control the test in a different manner and to perform additional operations as required. Most end users are comfortable working in a software environment that appears familiar and intuitive.

The concept of open source software is gaining popularity as users demand fair trade practices that do not confuse knowledge with technology. The GDS framework provides a unique opportunity to develop a vast collection of open source software that is bound to reduce the cost of at least routine testing and also benefit from the collective expertise of the testing community.

Conclusions. Changing circumstances in the global market force demand more affordable solutions that satisfy materials research, product development and quality assurance. Progress in this area is made possible with the appearance of lower-cost high performance components, modularization and increased levels of open source application software that permit the engineering of test equipment with reduced life cycle cost. Advances in this area will impact the further expansion of product development and manufacturing across an increasingly globalized world. 
Acknowledgments. The author deeply appreciates valuable inputs from partners and colleagues in the industry, R\&D and academia in the U.S., Russia, Ukraine, Malaysia and India many of whom shared the concerns that motivated the effort described in this paper. Ramesh Korradi provided some of the data for this review.

1. The Baldwin Testers: www.instron.com/wa/library/StreamFile.aspx? $\mathrm{doc}=466$.

2. History of the Dual-Stage Valve: http://www.moog.com/media/1/Design ofEHValveTechpaper.pdf.

3. J. C. Jones, Developments in Design of Electrohydraulic Control Valves from Their Initial Design Concept to Present Day Design and Applications, Workshop on Proportional and Servovalves, Monash University, Melbourne, Australia (1997).

4. http://www.moog.com/Media/1/761seriesvalves.pdf.

5. Bosch-Rexroth AG Hydraulic Components Catalog Part Type 4WRPEH6 (2007), p. 785.

Received 11. 06. 2008 